\title{
Dynamics of counterpropagating waves in parametrically driven systems: dispersion vs. advection
}

\author{
Carlos Martel , José M. Vega , Edgar Knobloch \\ E.T.S.I. Aeronáuticos. Universidad Politécnica de Madrid. 28040 Madrid. Spain \\ Department of Physics. Universiny of California. Berkeley. CA 94720. USA
}

\begin{abstract}
The dynamics of parametrically driven counterpropagating waves in a one-dimensional extended nearly conservative annular system are described by two coupled. damped, parametrically driven nonlinear Schrödinger (NLS) equations with opposite transport terms due to the group velocity, and small dispersion. The system is characterized by two length scales defined by a balance between (a) forcing and dispersion (the dispersive scale). and (b) forcing and advection at the group velocity (the transport scale). Both are large compared to the basic wavelength of the pattern. The dispersive scale plays an important role in the structure of solutions arising from secondary instabilities of frequency-locked spatially uniform standing waves (SW), and manifests itself both in traveling pulses or fronts and in extended spatio-temporal chaos, depending on the signs of the dispersion coefficient and nonlinearity.
\end{abstract}

PACS: 47.20.Ky; 47.20.Ma; 47.35.+i; 47.54.+r: 05.45..In

Keywords: Parametric resonance; Counterpropagating waves: Weak dispersion: Faraday waves

\section{Introduction}

The problem of parametric generation of waves by an external oscillatory field has a wide range of applications in many different areas of physics: Langmuir waves in plasmas, magnetic fluids subjected to an oscillatory magnetic field, light propagation in optical fibers or gravity-capillary waves on the surface of a vertically vibrated layer of fluid. Small amplitude non-propagative modes of a nearly conservative system of this type with one spatially extended dimension are described by the parametrically driven, damped nonlinear Schrödinger (NLS) equation. This equation has been extensively studied in the literature (see, e.g. [1-3] and references therein). As the forcing amplitude is increased, the trivial solution (corresponding to a spatially homogeneous state) loses stability to frequency-locked standing waves (SW). With increasing force these lose stability to localized non-propagating solitary waves (i.e., spatially inhomogeneous SW) and finally, for sufficiently strong forcing, the system exhibits spatio-temporal chaos. 
In this paper we investigate a more complicated problem: an extended reflection-symmetric system in which two counterpropagating wavetrains are simultaneously excited by the parametric forcing. The main difference between this case and the one described above centers on the presence of the group velocity. The group velocity advects spatial inhomogeneities in opposite directions for the two counterpropagating waves, and for slowly modulated wavetrains its effect dominates over dispersion. Two different balances are then possible producing two different length scales in the problem, both large relative to the basic wavelength of the $\mathrm{SW}$. The first scale is the transport scale $\ell_{\mathrm{g}}$ and results from a balance between advection and forcing; the second scale is the dispersive scale $\ell_{\mathrm{d}} \ll \ell_{\mathrm{g}}$ and characterizes the balance between forcing and dispersion. The former situation arises in studies of nonlinear hyperbolic equations in which both diffusion and dispersion are absent [4-6] while the latter is familiar from studies of non-propagative systems described by a single NLS or CGL (complex Ginzburg-Landau) equations. The present paper is devoted to understanding the conditions under which the dynamics of the system are dominated by the former balance (with the dispersive scales playing only a passive role) or the latter, with dispersive scales that are active and dominate the behavior of the system. These notions are made more precise in the remainder of this section.

The situation just described arises in the excitation of gravity-capillary waves on the surface of an almost inviscid fluid by vertical vibration (the one-dimensional Faraday experiment) when the associated slow mean flows can be neglected $[5,7,8]$, and in nonlinear pulse propagation in fiber gratings $[9,10]$ where the parametric excitation arises as a result of a spatial resonance. The discussion that follows uses the terminology of the Faraday system. We consider a weakly dissipative system that is large (as compared with the basic wavelength of the instability) in one spatial direction, hereafter the $x$-direction, and invariant under translations $x \rightarrow x+c$ and reflection $x \rightarrow-x$. We assume that there is a uniform steady solution of the unforced problem $\mathbf{u}_{0}$ (the quiescent state in the Faraday experiment) whose infinitesimal perturbations,

$$
\mathbf{u}=\mathbf{u}_{0}+\varepsilon \mathbf{U} \mathrm{e}^{\mathrm{i} k x+\omega t}+\cdots, \quad|\varepsilon| \ll \mathbf{1},
$$

obey the (complex) dispersion relation $\omega=\omega(k)$ shown in Fig. 1. Here $k$ is the (real) wavenumber. In other words, we assume that in the absence of forcing these perturbations oscillate with a frequency of order unity and decay very slowly because of the small damping $\delta$. When a system of this type is parametrically forced with a small forcing amplitude $\mu$ and frequency $\omega_{\mathrm{f}}$ it responds at the subharmonic frequency $\omega_{0}=\omega_{\mathrm{f}} / 2$, and this frequency in turn selects the wavenumber $k_{0}$ of the response according to the dispersion relation, viz., $\omega_{0}=\operatorname{lm}\left(\omega\left(k_{0}\right)\right.$ ) (see Fig. 1).

The resulting response can be expressed as a superposition of two slowly modulated counterpropagating wavetrains with wavenumbers $\pm k_{0}$ and frequency $\omega_{0}$,

$$
\mathbf{u}=\mathbf{u}_{0}+A(x, t) \mathbf{U}_{k_{0}} \mathrm{e}^{i k_{0} x+i \omega_{0} t}+B(x, t) \mathbf{U}_{-k_{0}} \mathrm{e}^{-i k_{0} x+i \omega_{0} t}+\text { c.c. }+\cdots .
$$

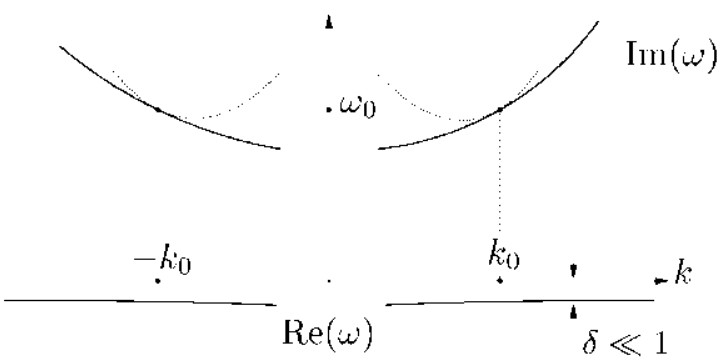

Fig. 1. Solid line: dispersion relation for infinitesimal perturbations of the basic state $\mathbf{u}_{0}$. Dotted line: dispersion relation of the zero state of the amplitude equations (4) and (5). 
Here $A$ and $B$ denote the scalar amplitudes of the two wavetrains and are assumed to vary slowly in both space and lime,

$$
\begin{aligned}
& \cdots \ll\left|A_{t}\right| \ll|A| \ll 1, \quad \cdots \ll\left|A_{x x}\right| \ll\left|A_{x}\right| \ll|A| \ll 1, \\
& \cdots \ll\left|B_{l}\right| \ll|B| \ll 1, \quad \cdots \ll\left|B_{x x}\right| \ll\left|B_{x}\right| \ll|B| \ll 1 .
\end{aligned}
$$

The weakly nonlinear cvolution of $A$ and $B$ is governed by the amplitude cquations $[5,7,11]$

$$
\begin{aligned}
& A_{t}=-\delta A+c_{\mathrm{g}} A_{x}+\mathrm{i} \alpha A_{x x}+\mathrm{i} A\left(n_{1}|A|^{2}+n_{2}|B|^{2}\right)+\mu \bar{B}+\cdots, \\
& B_{t}=-\delta B-c_{\mathrm{g}} B_{x}+\mathrm{i} \alpha B_{x x}+\mathrm{i} B\left(n_{1}|B|^{2}+n_{2}|A|^{2}\right)+\mu \bar{A}+\cdots,
\end{aligned}
$$

where the linear part results from a Taylor expansion of the dispersion relation $\omega(k)$ about $k= \pm k_{0}, \delta \ll 1$ is the damping, and $c_{\mathrm{g}} \sim 1$ and $\alpha \sim 1$ are, respectively, the group velocity and dispersion. The purely imaginary cubic terms represent a conservative nonlinearity and $\mu \ll 1$ is proportional to the forcing amplitude. In an annular domain of length $L \gg 1$ spatial periodicity implies that the boundary conditions for the amplitudes $A$ and $B$ are

$$
\Lambda(x+L, t) \mathrm{e}^{\mathrm{i} /}=\Lambda(x, t), \quad B(x+L, t) \mathrm{e}^{-\mathrm{i} /}=B(x, t),
$$

where $\theta=k_{0} L \bmod (2 \pi)$ measures the mismatch between the wavelength of the pattern and the domain period.

In the following we assume the primary balance $\delta \sim \mu \sim|A|^{2} \sim|B|^{2}$, cxpressing the requirement that the forcing must exced the effects of damping for an instability to occur, and that its nonlinear saturation determines the typical amplitude of the resulting pattern, and use $\delta$ as the common measure of all three. Under these circumstances Eqs. (4) and (5) define two distinct spatial scales: a dispersive scale, $\ell_{\mathrm{d}} \sim 1 / \sqrt{\delta} \gg 1$ obtained by balancing the forcing and dispersive terms, and a transport scale, $\ell_{\mathrm{g}} \sim 1 / \delta \gg 1$, obtained by balancing the advection term against the forcing. When $\delta \ll 1$ both scales are large compared with the basic wavelength of the wavetrains $2 \pi / k_{0} \sim 1$. Depending on the size of the domain $L \gg 1$ two different regimes are now possible [12] (see [13] for a similar discussion in the context of the oscillatory instability in dissipative systems):

- If $L \sim 1 / \sqrt{\delta}$ then $\ell_{\mathrm{d}} \sim L$ and $\ell_{\mathrm{g}} \sim L^{2}$, and hence $\ell_{\mathrm{g}}$ is 100 large, and only the dispersive scale fits in the domain. Then on the timescale $t \sim L$ the two amplitudes are uncoupled and simply travel at the group velocity in opposite directions in $x$. The remaining terms in Eqs. (4) and (5) affect the dy namics only on the much slower timescale $t \sim L^{2}$. Under these conditions it is possible to derive a simplificd system of equations from Eqs. (4) and (5) that describes the evolution of the amplitudes on this much slower lime scale. In this system the coupling terms appear spatially averaged reflecting the fact that, on this time scale, cach wave "sces" the other traveling very fast and therefore responds only to its average. This regime was analyzed in Ref. [12].

- If $L \sim 1 / \delta$ then $\ell_{\mathrm{g}} \sim L$ and $\ell_{\mathrm{d}} \sim L^{1 / 2} \ll \ell_{\mathrm{g}}$, and so both transport and dispersive scales (and all scales in between) can be present simultancously. In this case, characterized by the balance $1 / L \sim \delta \sim \mu \sim|A|^{2} \sim|B|^{2}$, no simplification is possible in general and the full system (4)-(6) must be considered. This is the regime investigated in this paper.

We begin by eliminating the parameter $\theta$ from the boundary conditions (6) using the change of variables

$$
(A, B)=\left(\tilde{A} \mathrm{e}^{-\mathrm{i} \theta x / L}, \tilde{B} \mathrm{e}^{\mathrm{i} \theta x / L}\right),
$$

and rescaling $x$ and $t$ (with $L$ and $L / c_{\mathrm{g}}$, respectively) to make both the system size and the group velocity equal to 1 . A rescaling of the amplitudes by the factor $\sqrt{c_{g} / L\left(n_{1}+n_{2}\right)}$ now yields the scaled equations (after dropping tildes)

$$
A_{t}-A_{x}=(-\delta+\mathrm{iv}) A+\mu \bar{B}+\mathrm{i} A\left(\beta|A|^{2}+(1-\beta)|B|^{2}\right)+\mathrm{i} \varepsilon A_{x x},
$$




$$
\begin{aligned}
& B_{t}+B_{x}=(-\delta+\mathrm{i} \nu) B+\mu \bar{A}+\mathrm{i} B\left(\beta|B|^{2}+(1-\beta)|A|^{2}\right)+\mathrm{i} \varepsilon B_{x x}, \\
& A(x+1, \imath)=A(x, \imath), \quad B(x+1, \ell)=B(x, \imath),
\end{aligned}
$$

where $\tilde{\delta}=\delta L / c_{\mathrm{g}} \sim 1, \tilde{\mu}=\mu L / c_{\mathrm{gg}} \sim 1,|\varepsilon|=\alpha / c_{\mathrm{g}} L \ll 1$ and $\beta=n_{1} /\left(n_{1}+n_{2}\right)$, with $\left.\left.\nu \in\right]-\pi, \pi\right]$ measuring the detuning between $\omega_{0}$ and the closest natural frequency of the system allowed by the periodic boundary conditions, $v \sim L\left(\omega-\omega_{0}\right)$. With this new scaling the transport scale is of order unity and the dispersive scale is of order $\sqrt{|\varepsilon|} \ll$ 1. Note that $n_{1}+n_{2}$ can always be made positive by taking. if necessary, complex conjugates of Eqs. (7)-(9).

Eqs. (7)-(9) have solutions that either develop structure on the dispersive scale $\ell_{\mathrm{d}}$ throughout the domain, or solutions in which the dominant balance involves only advection and nonlinearity, and the dispersive scale manifests itself at most locally. The solutions in the latter case are approximated to order $\varepsilon$ by the solutions of the following hyperbolic system

$$
\begin{aligned}
& A_{t}-A_{x}=(-\delta+\mathrm{i} v) A+\mu \bar{B}+\mathrm{i} A\left(\beta|A|^{2}+(1-\beta)|B|^{2}\right) \\
& B_{l}+B_{x}=(-\delta+\mathrm{i} \nu) B+\mu \bar{\Lambda}+\mathrm{i} B\left(\beta|B|^{2}+(1-\beta)|A|^{2}\right) \\
& A(x+1, t)=A(x, t), \quad B(x+1, t)=B(x, t) .
\end{aligned}
$$

This system of equations was derived in [5] for the particular case of capillary Faraday waves in deep containers, and used to conclude that almost uniform SW were the only attractors. In fact, as shown in Section 3, the dy namics with dispersion are much richer. Similar hy perbolic approximations for dissipative systems near a spontaneous Hopf bifurcation were considered by Daniels [4] in the context of rotating convection, and analyzed as approximations to the corresponding fully dissipative amplitude equations by two of us $[6,13]$ (see also [14] for a study of the resulting hyperbolic system). Fig. 2a shows a snapshot of a solution of Eqs. (7)-(9) that does not exhibit any structure on the dispersive scale, obtained for $\varepsilon=-10^{-4}$, together with the corresponding solution of the hyperbolic equations (10)(12); evidently for $\varepsilon=-10^{-4}$ these solutions are indistinguishable. In contrast, Fig. $2 \mathrm{~b}$ shows a solution of Eqs. (7)-(9) with structure on the smaller dispersive scale $\ell_{d}$ throughout the domain. Solutions of this type are not

(a)

$|A|, B \mid$
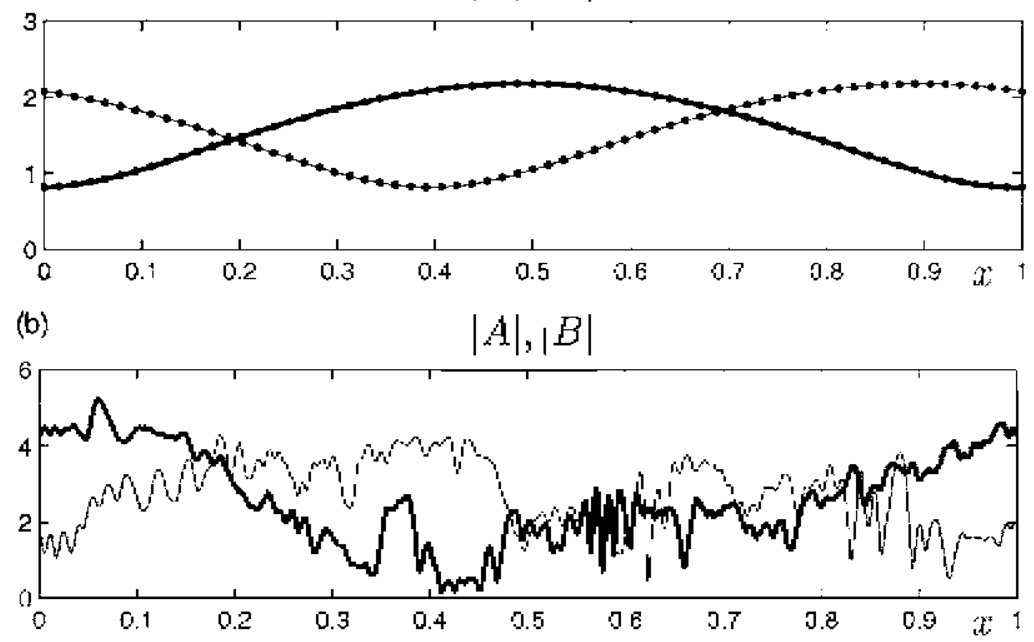

Fig. 2. (a) Steady solution of Fis. (7)-(9) for $\beta=2 / 3, \delta=1, v=0, \delta=-10^{4}$ and $\mu=3.04$, with spatial variation only on the transport scale. Dots: corresponding solution of the hyperbolic system (10)-(12). (b) A snapshot of a fully resolved solution of Ecs. (7)-(9) for lighler forcing, $\mu=4.0$, showing structure on the dispersive scale $(|A|$ : thick line, $|B|$ : thin line). 
contained in the hyperbolic approximation (10)-(12) and the inclusion of dispersion, however small, is essential. Thus no simplifications of Eqs. (7)-(9) are possible.

The remainder of the paper is organized as follows. In the next section we study the simplest solutions of Eqs. (7)-(9), namely spatially uniform SW, and their lincar stability properties. In Section 3, we present numerical solutions of Eqs. (7)-(9) corresponding to the various regimes identificd in Section 2, focusing on the consequences of changing the nonlinear coefficient $\beta$ and the sign of the dispersion coefficient $\varepsilon$. These results indicate that in almost all cases the instability of the spatially uniform SW cvolves into a state with complex spatio-lemporal dynamics, cxhibiting all spatial scales $\ell, \ell_{\mathrm{d}}<\ell<\ell_{\mathrm{g}}$. The paper concludes with a bricf discussion.

\section{Stability properties of SW}

The zero solution of Eqs. (7)-(9), viz. $(A, B)=(0,0)$, is stable against perturbations of the form $\left(a^{k}, b^{k}\right) \mathrm{e}^{\mathrm{i} 2 \pi k+s t}$ provided that the forcing amplitude does not exceed the critical value $\mu_{0}$, given by

$$
\mu_{0}=\sqrt{\delta^{2}+(v+2 \pi k)^{2}},
$$

up to $\sqrt{|\varepsilon|}$ corrections. This equation describes the typical instability tongues produced by parametric resonance (Fig. 3). The first mode to become unstable is that with $k=0$ and if the forcing is further increased the nearby modes are also destabilized.

At $\mu=\mu_{0}$ a branch of steady solutions bifurcates from the zero state. These steady solutions are of the form

$$
A_{0}=R_{0} \mathrm{c}^{\mathrm{i} \theta_{0}+\mathrm{i} 2 \pi m x}, \quad B_{0}=R_{0} \mathrm{c}^{\mathrm{i} \partial_{0}-\mathrm{i} 2 \pi m x}
$$

with $R_{0} \geq 0$ and $\theta_{0}$ given by (at leading order in $\varepsilon$ )

$$
\begin{aligned}
& {\left[R_{0}^{2}+v+2 \pi m\right]^{2}+\delta^{2}=\mu^{2},} \\
& \cos 2 \theta_{0}=\frac{\delta}{\mu}, \quad \sin 2 \theta_{0}=\frac{R_{0}^{2}+v+2 \pi m}{\mu},
\end{aligned}
$$

where $m \in \mathbb{Z}$. In terms of the original physical variable $\mathbf{u}(x, t)$, Eq. (1), this family of solutions corresponds to spatially uniform frequency-locked SW with $m$ more wavelengths in $0<x<L$ than the reference wavetrain. The corresponding bifurcation is supercritical (subcritical) if $\tilde{v}>0(\tilde{v}<0)$, and in the supercritical case the first SW to appear is that with $m=0$ (Fig. 4a). Here $\bar{v} \equiv v+2 \pi m$ may be called the extended detuning. It is convenient to represent the resulting SW solutions in the $\left(\tilde{v}, R_{0}^{2}\right)$ plane. Each point of this plane corresponds to a single $\mathrm{SW}$ and vice versa, and there are no multiple solutions (Fig. 4b).

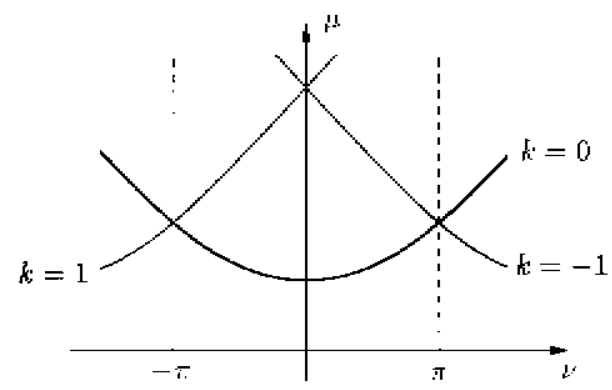

Fig. 3. Stability diagram of $A=B=0, v \in\rceil-\pi, \pi]$ (shading indicales instability). 

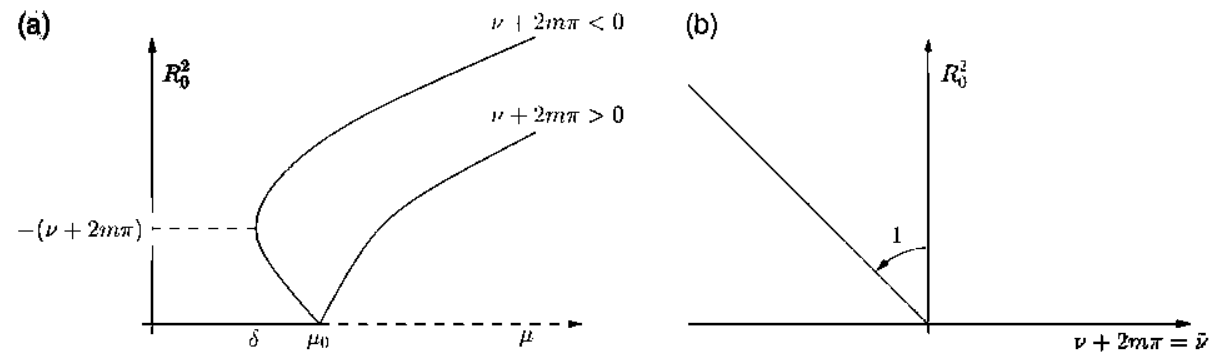

Fig. 4. (a) Sketch of the two possible SW bifurcation diagrams. (b) The $\left(\tilde{\nu}, R_{0}^{2}\right)$ representation of the SW (shading indicates the lower part of the subcritical branch).

Infinitesimal perturbations of the SW solutions, defined by

$$
A=A_{0}(1+a), \quad B=B_{0}(1+b),
$$

satisfy the linearized equations

$$
\begin{aligned}
& a_{t}-a_{x}=(-\delta+\mathrm{i} \bar{v})(a-\bar{b})+\mathrm{i} \beta R_{0}^{2}((a+\bar{a})-(b+\bar{b}))+\mathrm{i} R_{0}^{2}(a+b)+\mathrm{i} \varepsilon a_{x x}, \\
& b_{t}+b_{x}=(-\delta+\mathrm{i} \tilde{v})(b-\bar{a})-\mathrm{i} \beta R_{0}^{2}((a+\bar{a})-(b+\bar{b}))+\mathrm{i} R_{0}^{2}(a+b)+\mathrm{i} \varepsilon b_{x x}, \\
& a(x+1, t)=a(x, t), \quad b(x+1, t)=b(x, t) .
\end{aligned}
$$

This system is solved via the Fourier expansion

$$
(a, b)=\sum_{k=-\infty}^{\infty}\left(a^{k}(t), b^{k}(t)\right) \mathrm{e}^{\mathrm{i} 2 \pi k x},
$$

whose coefficients satisfy

$$
\begin{aligned}
& a_{t}^{k}=(-\delta+\mathrm{i} \tilde{\nu})\left(a^{k}-\bar{b}^{-k}\right)+\mathrm{i} \beta R_{0}^{2}\left(\left(a^{k}+\bar{a}^{-k}\right)-\left(b^{k}+\bar{b}^{-k}\right)\right)+\mathrm{i} R_{0}^{2}\left(a^{k}+b^{k}\right)-\mathrm{i}\left(\varepsilon(2 \pi k)^{2}-(2 \pi k)\right) a^{k}, \\
& b_{t}^{k}=(-\delta+\mathrm{i} \tilde{\nu})\left(b^{k}-\bar{a}^{-k}\right)-\mathrm{i} \beta R_{0}^{2}\left(\left(a^{k}+\bar{a}^{-k}\right)-\left(b^{k}+\bar{b}^{-k}\right)\right)+\mathrm{i} R_{0}^{2}\left(a^{k}+b^{k}\right)-\mathrm{i}\left(\varepsilon(2 \pi k)^{2}+(2 \pi k)\right) b^{k} .
\end{aligned}
$$

For each $k$ these equations together with their complex conjugates form a quartet that is uncoupled from the corresponding equations for the other wavenumbers. Solutions proportional to $\mathrm{e}^{\Omega 2 l}$ satisfy a fourth-order dispersion relation for the eigenvalues $\Omega$. The discussion of the resulting stability problem is easier if the perturbations with $(k \sim 1 / \sqrt{|\varepsilon|})$ and without $(k \sim 1)$ small dispersive scales are studied separately.

For perturbations with wavenumber of order unity (i.e., without dispersive scales) the dispersive terms can be neglected and the resulting equation for $\Omega$ is

$$
\Omega(\Omega+2 \delta)\left(\Omega(\Omega+2 \delta)+2(2 \pi k)^{2}+4 R_{0}^{2}\left(\tilde{v}+R_{0}^{2}\right)\right)+(2 \pi k)^{4}-4(2 \pi k)^{2}\left(\tilde{\nu}+R_{0}^{2}\right)\left(\tilde{v}+2 \beta R_{0}^{2}\right)=0 .
$$

For the particular case of spatially uniform perturbations, $k=0$, the solutions of (22) are

$$
\Omega=0, \quad \Omega=-2 \delta, \quad \Omega=-\delta \pm \sqrt{\delta^{2}-4 R_{0}^{2}\left(\tilde{v}+R_{0}^{2}\right)},
$$

where the \%ero eigenvalue comes from the invariance of Eqs. (7)-(9) under rotations $(A, B) \rightarrow\left(A \mathrm{c}^{\mathrm{i} \alpha}, B \mathrm{c}^{-\mathrm{i} \alpha}\right)$, $\alpha \in \mathbb{R}$, corresponding to spatial translations in the original physical system, sec Eq. (I). Evidently, the SW are 

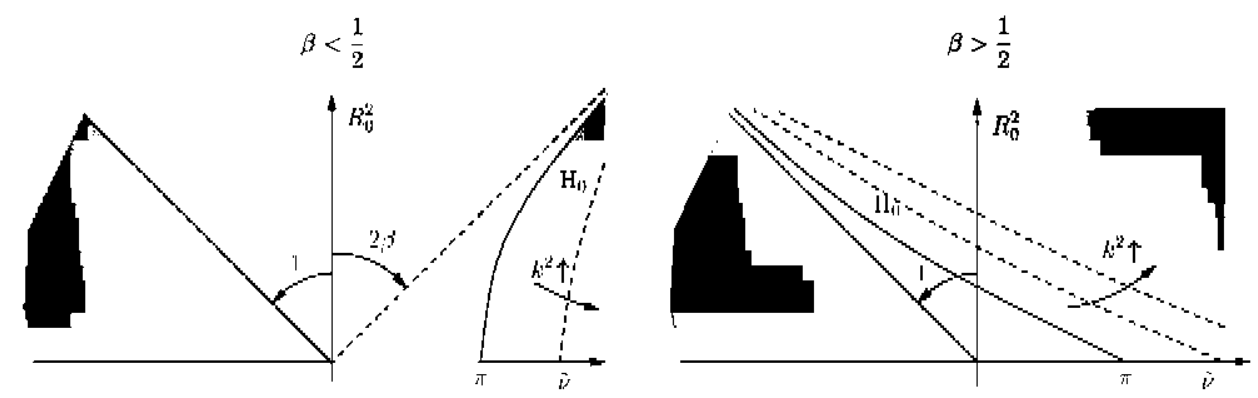

Fig. 5. Solid line: the hyperbola $\mathrm{Ii}_{0}$ given by $\mathrm{Eq}$. (24), with shading indicating instability. Dashed lines: condition (23) lor different values of $k^{2}$.

unstable against spatially uniform perturbations if $\tilde{v}+R_{0}^{2}<0$, i.e., along the lower part of the SW branch when $\tilde{v}<0$ (see Fig. $4 \mathrm{~b}$ ). In the following we assume therefore that $\tilde{v}+R_{0}^{2}>0$.

Perturbations with wavenumber $k \neq 0$ become unstable through a steady bifurcation $(\Omega=0$ ) when entering the region

$$
\left(\bar{v}+R_{0}^{2}\right)\left(\bar{v}+2 \beta R_{0}^{2}\right)-(\pi k)^{2}>0 .
$$

The modes that first become unstable are always those with $k^{2}=1$; when all modes are taken into account the instability region lies on the positive side of the hyperbola $\left(\mathrm{H}_{0}\right)$

$$
\pi^{2}-\left(\tilde{v}+R_{0}^{2}\right)\left(\tilde{v}+2 \beta R_{0}^{2}\right)=0,
$$

see Fig. 5.

In contrast, purely imaginary eigenvalues occur along

$$
\left(\tilde{v}+R_{0}^{2}\right)\left[\tilde{v}+(2 \beta+1) R_{0}^{2}\right]+\delta^{2}+\frac{R_{0}^{2}\left(\tilde{v}+R_{0}^{2}\right)\left[2 \delta^{2}+R_{0}^{2}\left(\tilde{v}+R_{0}^{2}\right)\right]}{(2 \pi k)^{2}}=0,
$$

and modes with wavenumber $k$ are stable for positive values of this expression and unstable otherwise. When the SW are stable against uniform perturbations, i.c., $\tilde{v}+R_{0}^{2}>0$, these curves exist only if $\beta<0$, and all are then enclosed within the hyperbola $\left(\mathrm{H}_{\mathrm{i}}\right)$

$$
\delta^{2}+\left(\tilde{v}+R_{0}^{2}\right)\left\lfloor\tilde{v}+(2 \beta+1) R_{0}^{2}\right\rfloor=0 .
$$

This line marks another stability limit for the SW (sec Fig. 6); above this line growing perturbations are always present provided their wavenumber is sufficiently large.

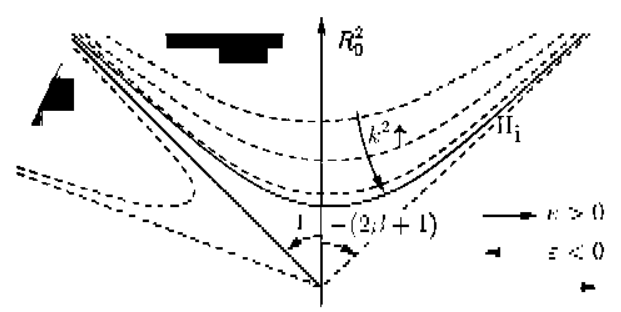

Fig. 6. Solid line: the hyperbola $\mathrm{H}_{i}$ given by Fq. (26) for $\beta<0$, with shading indicating instability. Jashed lines: condition (25) for inereasing values of $k^{2}$. The solid line in the subcritical region corresponds to $\mathrm{II}_{\mathrm{l}}$ for $\beta \approx 0$, the high wavenumber instability condition (27) is obtained by shifting $\mathrm{H}_{\mathrm{i}}$ in the direction ol the arrows. 
A stability condition similar to (26) is obtained by examining the dispersion relation in the high wavenumber regime, i.c., on the dispersive scalc $|k| \sim 1 / \sqrt{|\varepsilon|} \gg 1$. To this end we set $\kappa=(2 \pi k) \sqrt{|\varepsilon|} \sim 1$ and expand the solution of Eqs. (17) and (18) in the form

$$
a^{\kappa}=a_{0}^{\kappa}(l, T)+\sqrt{|\varepsilon|} a_{1}^{\kappa}(l, T)+\cdots, \quad b^{\kappa}=b_{0}^{\kappa}(l, T)+\sqrt{|\varepsilon|} b_{1}^{\kappa}(l, T)+\cdots,
$$

where $T=t / \sqrt{|\varepsilon|}$. At first order we obtain

$$
\frac{\partial a_{0}^{\kappa}}{\partial T}-\mathbf{i} \kappa a_{0}^{\kappa}=0, \quad \frac{\partial b_{0}^{\kappa}}{\partial T}+\mathbf{i} \kappa b_{0}^{\kappa}=0,
$$

whose general solution is

$$
\left(a_{0}^{\kappa}, b_{0}^{\kappa}\right)=\left(A_{0}^{\kappa}(t) \mathrm{e}^{\mathrm{i} \kappa T}, B_{0}^{\kappa}(t) \mathrm{e}^{-\mathrm{i} \kappa T}\right) .
$$

Thus, on the fast time scale $T$, these high wavenumber perturbations simply travel in opposite directions at the group velocity. At next order we obtain

$$
\begin{aligned}
\frac{\partial a_{1}^{\kappa}}{\partial T}-\mathrm{i} \kappa a_{1}^{\kappa}= & {\left[-A_{0 t}^{\kappa}+\left(-\delta+\mathrm{i}\left(\tilde{v}+(\beta+1) R_{0}^{2}-\frac{\varepsilon}{|\varepsilon|} \kappa^{2}\right)\right) A_{0}^{\kappa}+\mathrm{i} \beta R_{0}^{2} \bar{A}_{0}^{-\kappa}\right] \mathrm{e}^{\mathrm{i} \kappa T} } \\
& -\left[\left(-\delta+\mathrm{i}\left(\tilde{v}+\beta R_{0}^{2}\right)\right) \bar{B}_{0}^{-\kappa}+\mathrm{i}(\beta-1) R_{0}^{2} B_{0}^{\kappa}\right] \mathrm{e}^{-\mathrm{i} \kappa T}, \\
\frac{\partial b_{1}^{\kappa}}{\partial T}+\mathrm{i} \kappa b_{1}^{\kappa}= & {\left[-B_{0 t}^{\kappa}+\left(-\delta+\mathrm{i}\left(\tilde{v}+(\beta+1) R_{0}^{2}-\frac{\varepsilon}{|\varepsilon|} \kappa^{2}\right)\right) B_{0}^{\kappa}+\mathrm{i} \beta R_{0}^{2} \bar{B}_{0}^{-\kappa}\right] \mathrm{e}^{-\mathrm{i} \kappa T} } \\
& -\left[\left(-\delta+\mathrm{i}\left(\tilde{v}+\beta R_{0}^{2}\right)\right) \bar{A}_{0}^{-\kappa}+\mathrm{i}(\beta-1) R_{0}^{2} A_{0}^{\kappa}\right] \mathrm{e}^{\mathrm{i} \kappa T} .
\end{aligned}
$$

The solvability conditions for these two equations (i.e., the conditions that ensure that $\left(a_{1}^{\kappa}, b_{1}^{k}\right)$ will remain bounded in the fast time scale $T)$ give the equations for the evolution of $\left(A_{0}^{\kappa}, B_{0}^{\kappa}\right)$ on the timescale $t$ :

$$
\begin{aligned}
& \frac{\mathrm{d} \Lambda_{0}^{\kappa}}{\mathrm{d} t}=\left(-\delta+\mathrm{i}\left(\tilde{y}+(\beta+1) R_{0}^{2}-\frac{\varepsilon}{|\varepsilon|} \kappa^{2}\right)\right) A_{0}^{\kappa}+\mathrm{i} \beta R_{0}^{2} \bar{A}_{0}^{-\kappa}, \\
& \frac{\mathrm{d} B_{0}^{\kappa}}{\mathrm{d} t}=\left(-\delta+\mathrm{i}\left(\tilde{y}+(\beta+1) R_{0}^{2}-\frac{\varepsilon}{|\varepsilon|} \kappa^{2}\right)\right) B_{0}^{\kappa}+\mathrm{i} \beta R_{0}^{2} \bar{B}_{0}^{-\kappa} .
\end{aligned}
$$

The short wave counterpropagating perturbations are thus uncoupled with identical eigenvalues

$$
s=-\delta \pm \sqrt{\beta^{2} R_{0}^{4}-\left[\tilde{v}-\frac{\varepsilon}{|\varepsilon|} \kappa^{2}+(\beta+1) R_{0}^{2}\right]^{2}},
$$

and hence are unstable if

$$
\delta^{2}+\left(\tilde{v}-\frac{\varepsilon}{|\varepsilon|} \kappa^{2}+R_{0}^{2}\right)\left(\tilde{v}-\frac{\varepsilon}{|\varepsilon|} k^{2}+(2 \beta+1) R_{0}^{2}\right) \leq 0 .
$$

This instability criterion is identical to (26) except for a shift in the detuning of size $\kappa^{2}$. A variation in wavenumber from $k$ to $k+1$ produces a very small increment of $k, \Delta \kappa \sim \sqrt{|\varepsilon|} \ll 1$, so the resulting instability region defined by condition (27) is just the envelope of the translations of the curve defined by Eq. (26) towards positive $\tilde{v}$ if $\varepsilon>0$ and towards negative $\tilde{v}$ otherwise (Fig. 6). Condition (27) is essentially the Benjamin-Feir instability condition for the Stokes wave in the NLS equation (sec, e.g. $[15,16]$ ) but generalized to include the effects of damping, detuning and nonlinear cross-coupling. It is important to observe that in the presence of small dispersion and diffusion, i.c., for Eqs. (10) and (11) with $(|\varepsilon| \eta+\mathrm{i} \varepsilon) A_{x x}$ and $(|\varepsilon| \eta+\mathrm{i} \varepsilon) B_{x x}(\eta>0)$ instcad of 
$\mathrm{i} \varepsilon A_{x x}$ and i $\varepsilon B_{x x}$ on the right-hand side, the high wavenumber instability condition (27) remains valid provided $\delta$ is replaced by $\delta+\eta \kappa^{2}$. It follows that the addition of diffusion always delays the onset of the small scale instability. In the extreme case in which dispersion is absent all instability is confined within the curve $\mathrm{H}_{\mathrm{i}}$ defined by Eq. (26).

In Fig. 7, we summarize the final stability diagrams for the $\mathrm{SW}$ for different values of the nonlinear coefficient $\beta$ and signs of the dispersion coefficient $\varepsilon$. The SW are always stable near onset when the bifurcation is supercritical, that is, when the detuning is positive (Fig. 4). However, the sign of the dispersion changes drastically the stability properties of the resulting SW. If the dispersion is positive (Fig. $7 \mathrm{I}^{+}, \mathrm{II}^{+}, \mathrm{III}^{+}$and $\mathrm{IV}^{+}$) almost all SW with amplitude
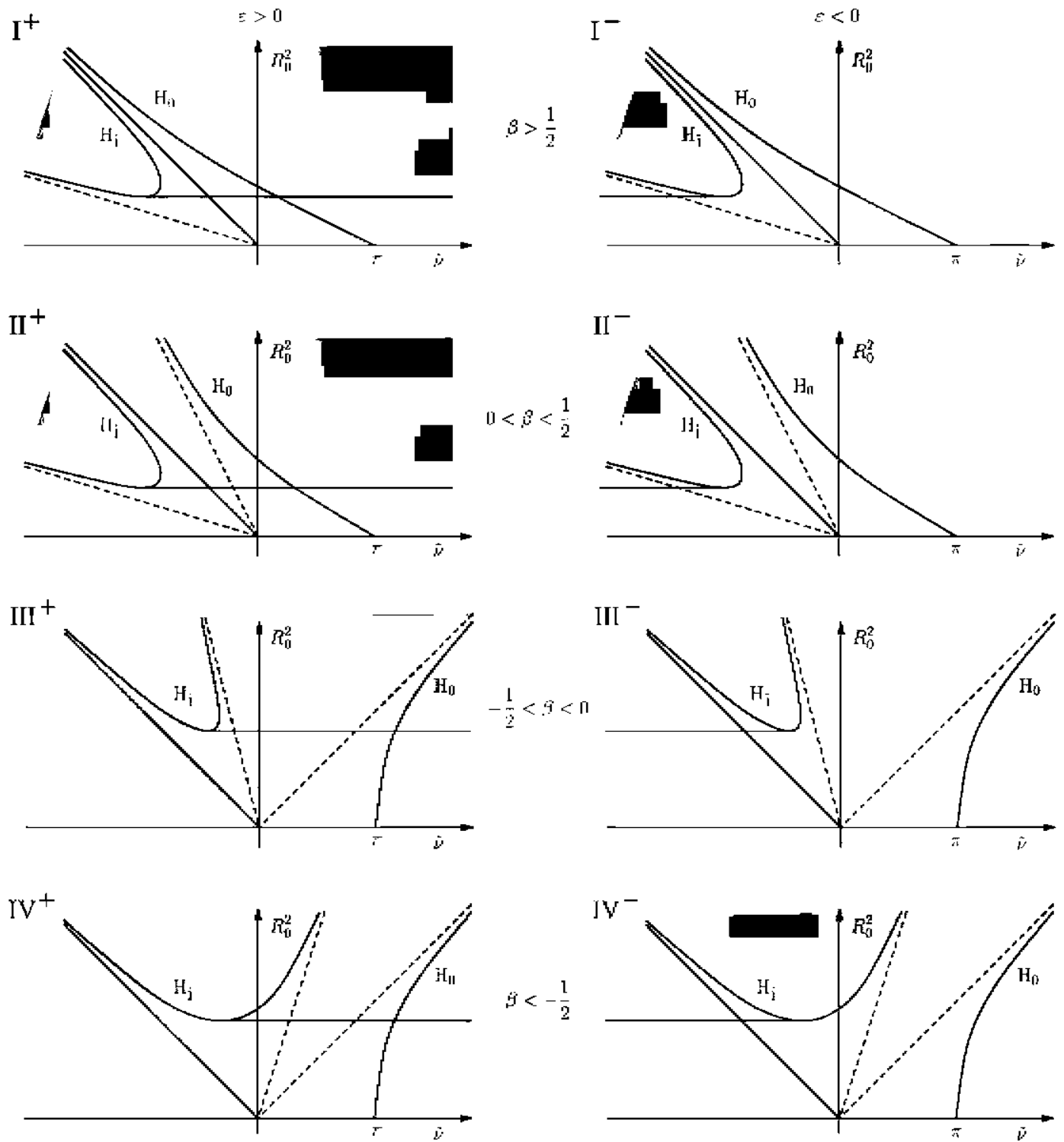

Fig. 7. Stability properties of the SW, with light (dark) shading indicating long (short) wave instability. 
above the minimum of $\mathrm{H}_{\mathrm{i}}$,

$$
R_{\min }=\sqrt{\frac{\delta}{|\beta|}},
$$

become unstable against perturbations containing dispersive scales. When $\varepsilon<0$ and $\beta>0$, the instability that occurs with increasing forcing is a steady bifurcation (line $\mathrm{H}_{0}$ in Fig. $7 \mathrm{I}^{-}$and $\mathrm{II}^{-}$) and the modes that are destabilized are long waves: just one wavelength per spatial period. The SW remain stable for all values of the forcing only in the case $\varepsilon<0,-1 / 2<\beta<0$ (Fig. 7III-) and $0<\tilde{\nu}<\pi$, and they lose and regain stability as $R_{0}^{2}$ (i.e., the forcing) increases if $\tilde{v}<\tilde{v}_{\max }=-\delta \sqrt{2 \beta+1} /|\beta|<0$. Finally, if $\varepsilon<0$ and $\beta<-1 / 2$ (Fig. 7IV ${ }^{-}$) high wavenumber perturbations always destabilize the SW if the forcing is large enough. Thus in the case $\varepsilon<0$ the small scale instability has, in general, a much less dramatic effect.

The dispersive instability, given by condition (27), takes place closer and closer to the onset of the SW as the damping $\delta$ decreases (see Eq. (28)); if $\nu$ and $\mu$ are also small, the system is in the first regime of Section 1 and the stability results of [12] are recovered.

\section{Complex dynamics}

In this section we discuss the consequences of the different types of instability identified in the preceding section, and relate the results to the question raised in Section 1 . The section is largely numerical since analytical techmiques do not permit us to completely characterize the dynamics that result unless the system is very close to one of the possible secondary instabilities. The numerical integrations of Eqs. (7)-(9) were performed using a Fourier series expansion in space with $N_{\text {Fourier }}$ modes and a fourth-order Runge-Kutta scheme for the time integration of the resulting system of ODEs. The linear terms (damping, detuning, group velocity and dispersion) were integrated implicitly, typically using $N_{\text {Fourier }}=2048$ with a time step $\Delta t=0.0001$.

We focus on solutions in the eight different regimes identified in Fig. 7, but make no attempt at an exhaustive exploration of the (five-dimensional) parameter space of Eqs. (7)-(9). The results described below suffice to give an idea of the rich variety of solutions that appear when the SW lose stability, and how these change depending on dispersion and nonlinearity.

We begin with the case $\beta=2 / 3, \delta=1, \nu=0$ and $\varepsilon=10^{-4}$ (Fig. $7 \mathrm{I}^{+}$). The SW are stable if the forcing is below the critical value $\mu_{\mathrm{c}} \cong 1.80$, given by Eqs. (15) and (28). At this point an instability against high wavenumber perturbations sets in supercritically and produces modulation of the SW in the form of a pair of counterpropagating wavetrains with small (dispersion-dominated) wavelength $\lambda \sim \sqrt{|\varepsilon|} \ll 1$. These wavetrains travel with the group velocity and remain spatially periodic with a small spatial period (Fig. 8). In terms of the original physical variable $\mathbf{u}(x, t), \mathrm{Eq} .(1)$, the resulting state of the system consists of small regions (i.e., of size $L^{1 / 2}$ ) in which the left- and right-traveling waves alternately dominate. When the forcing is increased beyond $\mu \cong 3.05$ the solution starts to develop spatial modulations on the scale of the total size of the domain (i.e., of size $L$ ). A1 $\mu \cong 3.25$, there is a Iransition via intermittency to a new branch of solutions in which the spatial periodicity on the dispersive scale is completely lost. The solution for $\mu=4$ is shown in Fig. 9. Notice that, for short times, the small dispersive scales are largely advected at the group velocity, but also evolve on a slower timescale, $t \sim 1$, to produce very complicated spatio-temporal dynamics.

The situation found for $\beta=1 / 3, \delta=1, v=0$ and $\varepsilon=10^{-4}$ (Fig. $71 \mathrm{I}^{+}$) is rather similar to that of the previous case: the SW are stable up to $\mu_{\mathrm{c}} \cong 3.16$ (sec Eqs. (15) and (28)), where spatially periodic traveling waves with small period develop supercritically and then, at $\mu \cong 3.83$, there is an abrupt transition to a branch of spatio-temporally 


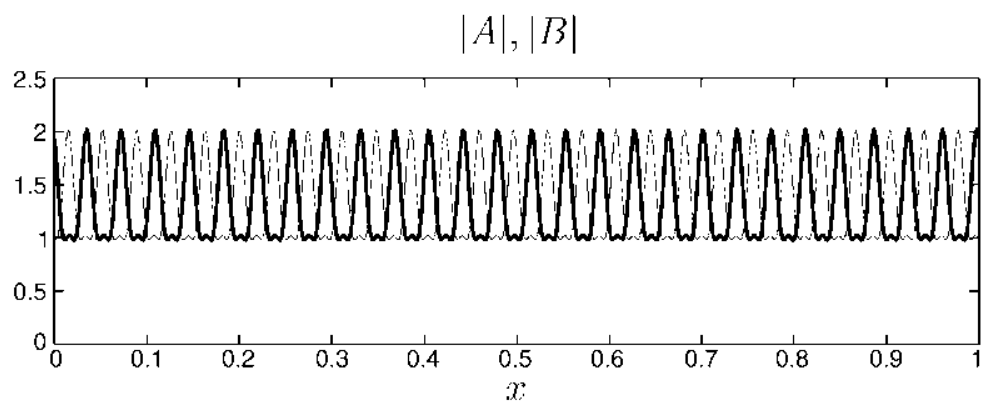

Fig. 8. Solution of Eqs. (7)-(9) for $\beta=2 / 3, \delta=1, v=0, \varepsilon=10^{-4}$ and $\mu=2.5$, at a particular instant in time. $|A|$ (thick linc) travels to the left and $|B|$ (thin line) to the right. The modulation length scale is $\mathcal{O}\left(|\varepsilon|^{1 / 2}\right.$ ).
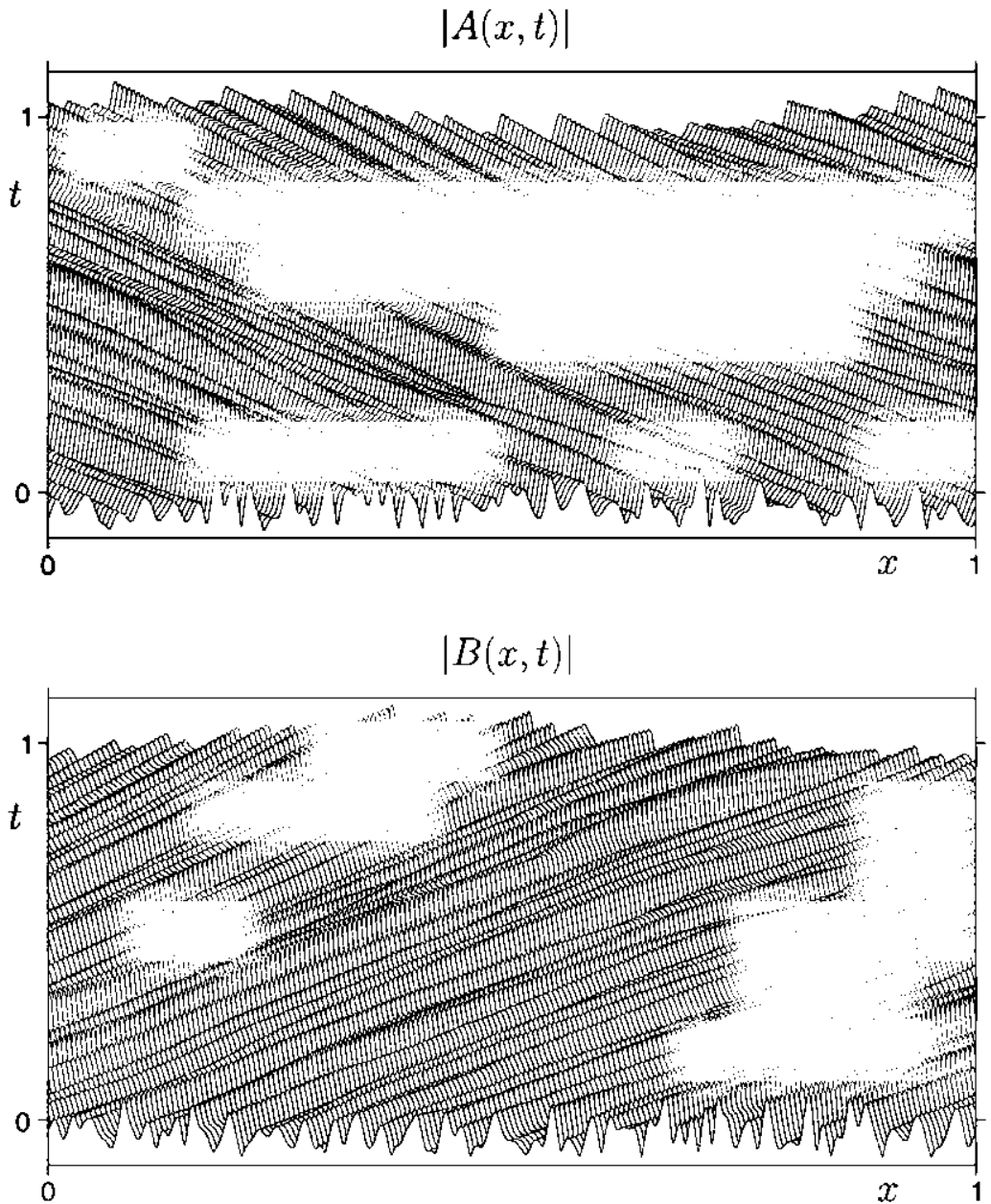

Fig. 9. Spacc-time representation of the solution of Eqs. (7)-(9) for $\beta=2 / 3, \delta=1, v=0, \varepsilon=10^{-4}$ and $\mu=4$. 

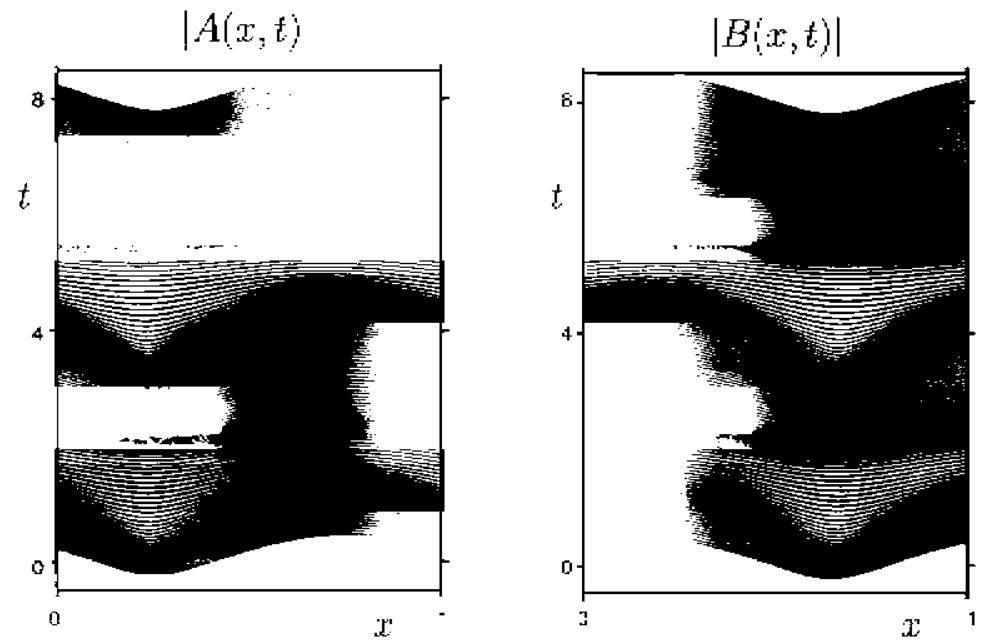

Fig. 10. Space-time representation of a temporally chaotic solution of H.qs. (7)-(9) for $\beta=2 / 3, \delta=1, v=0,8=-10^{4}$ and $\mu=3.10$, showing large scale oscillations with no dispersive scales present.
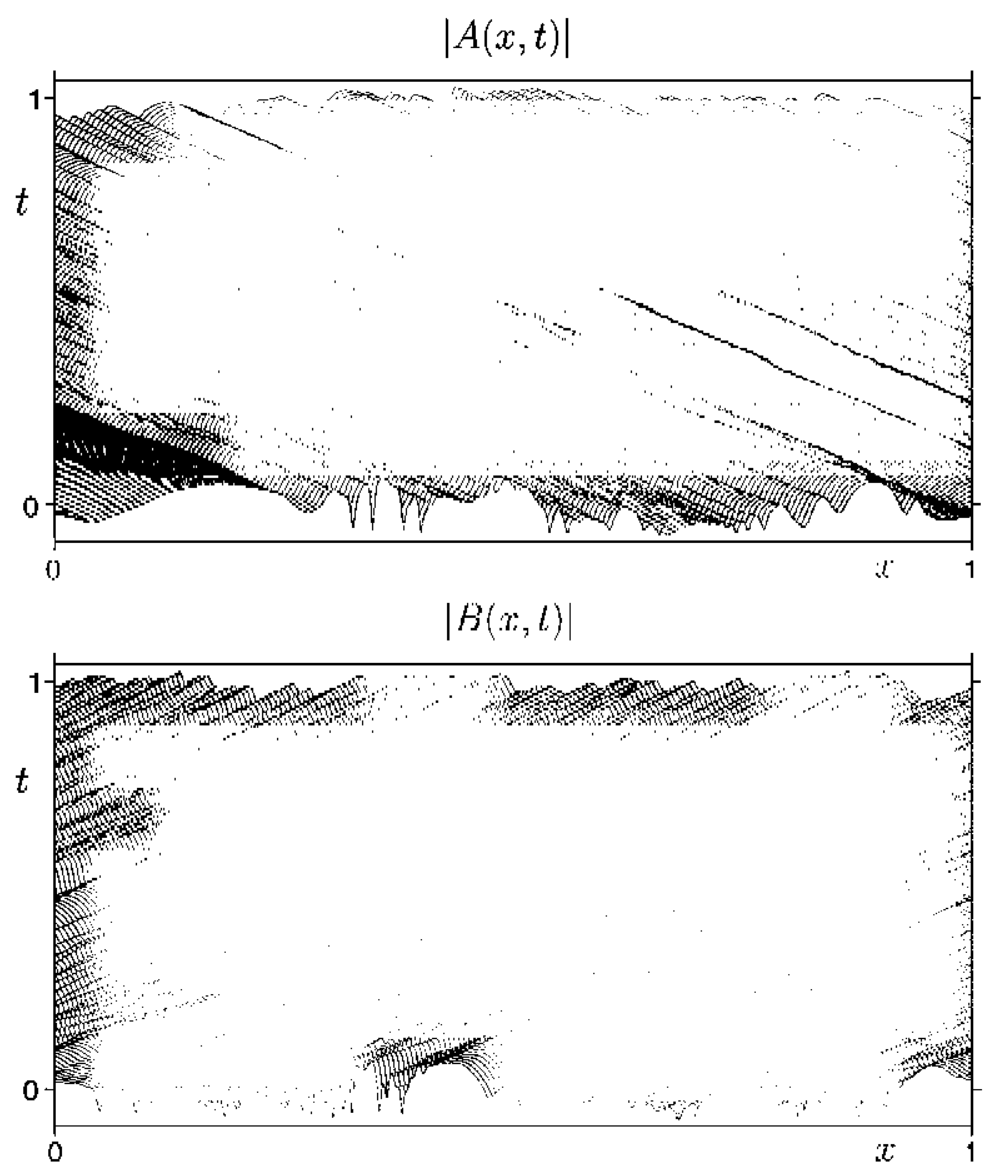

Fig. 11. Space-time representation of the solution of Egs. (7)-(9) for $\beta=2 / 3, \delta=1, v=0, s=-10^{-4}$ and $\mu=4$. 
complex solutions. The main difference between this and the previous case is that this last transition is now hysteretic and the new branch of complex solutions persists down to $\mu \cong 2.25$ before stable SW are recovered.

When the dispersion is negative (case $\beta=2 / 3, \delta=1, v=0$ and $\varepsilon=-10^{-4}$, Fig. $7 \mathrm{I}^{-}$) the destabilization of the SW takes a completely different form: the SW lose stability to perturbations with wavenumber $k=1$ at $\mathrm{H}_{0}$ corresponding to $\mu_{\mathrm{c}} \cong 2.90$ (obtained from Eqs. (15) and (24)). The solutions that appear for $\mu>\mu_{\mathrm{c}}$ are stcady, non-uniform but rellection-symmetric, and without small dispersive scales (sec Fig. 2a). The reconstructed solution $\mathbf{u}(x, t)$, Eq. (1), consists of right-traveling waves filling one half of the annular domain and left-traveling waves filling the other half. For higher values of the forcing these solutions become oscillatory through a Hopf bifurcation and then chaotic after a sequence of period doublings but still without dispersive scales (Fig. 10); all these solutions can be computed accurately (to within $\mathcal{O}(\varepsilon)$ ) using the hyperbolic system (10)-(12). Dispersive scales appear in the solution only at $\mu \cong 3.11$ producing a disordered state filling the entire spatial domain. Thus the hyperbolic approximation (10)-(12) describes correctly the behavior of the system in the range $2.90<\mu<3.11$, but fails for
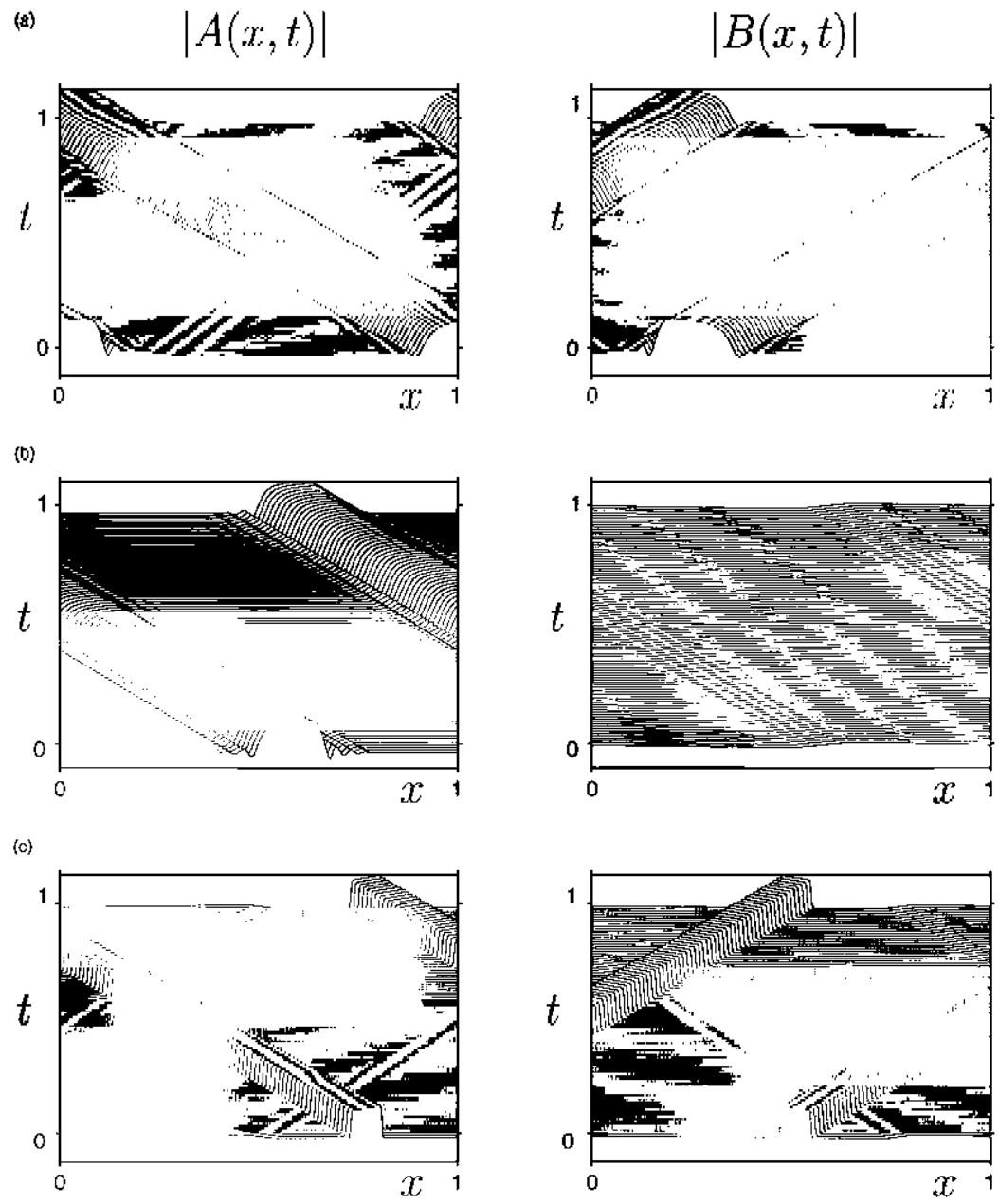

Fig. 12. Space time representation of the solution of Eqs. (7) (9) for $\beta=-1, \delta=1, \nu=1, \varepsilon=10^{-4}$ and (a) $\mu=2.25$ and (b) $\mu=2.10$. (c) Space time representation of the solution of the hyperbolic system (10) (12) for $\beta=-1, \delta=1, \nu=1$ and $\mu=2.25$ (with small diffusion $10{ }^{6}$ added to smooth the fronts). 
larger values of $\mu$. An cxample of the complex solutions that are produced at these larger values of $\mu$ is shown in Fig. 11 for $\mu=4$.

In the $\operatorname{case} \beta=1 / 3, \delta=1, v=1$ and $\varepsilon=-10^{-4}$ (Fig. 711-) the dynamics of the system appear to be very simple: the solutions found in our numerical simulations are always stable $\mathrm{SW}$. When the forcing is increased bey ond the line $\mathrm{H}_{0}$ there is an Eckhaus-type instability and the system simply selects a new branch of stable SW with one less wavelength.

When the nonlinear coefficient is negative, $\beta=-1, \delta=1, v=1$ and $\varepsilon=10^{-4}$ (Fig. 7IV ${ }^{+}$), the SW branch bifurcates from the zero solution at $\mu=\mu_{0} \cong 1.41$, sec Eq. (13), and remains stable for $\mu<\mu_{\mathrm{o}} \cong 2.24$ (sec Eqs. (15) and (28)). At this point the system undergoes a hysteretic transition to a new state in which the small dispersive scales are confined to thin layers connecting intervals with no dispersive scales. Fig. 12a shows such a (symmetric) state when $\mu=2.25$. The 'pulse'-like structure in each amplitude travels with the group velocity, and produces a solution $\mathbf{u}(x, t)$, Eq. (1), that is made up of traveling patches of traveling waves that propagate over a background of non-zero SW. When the forcing amplitude is reduced asymmetric solutions, in which a pulse forms in one of the amplitudes only, are found (Fig. 12b). These pulse-like solutions or bound states are contained in
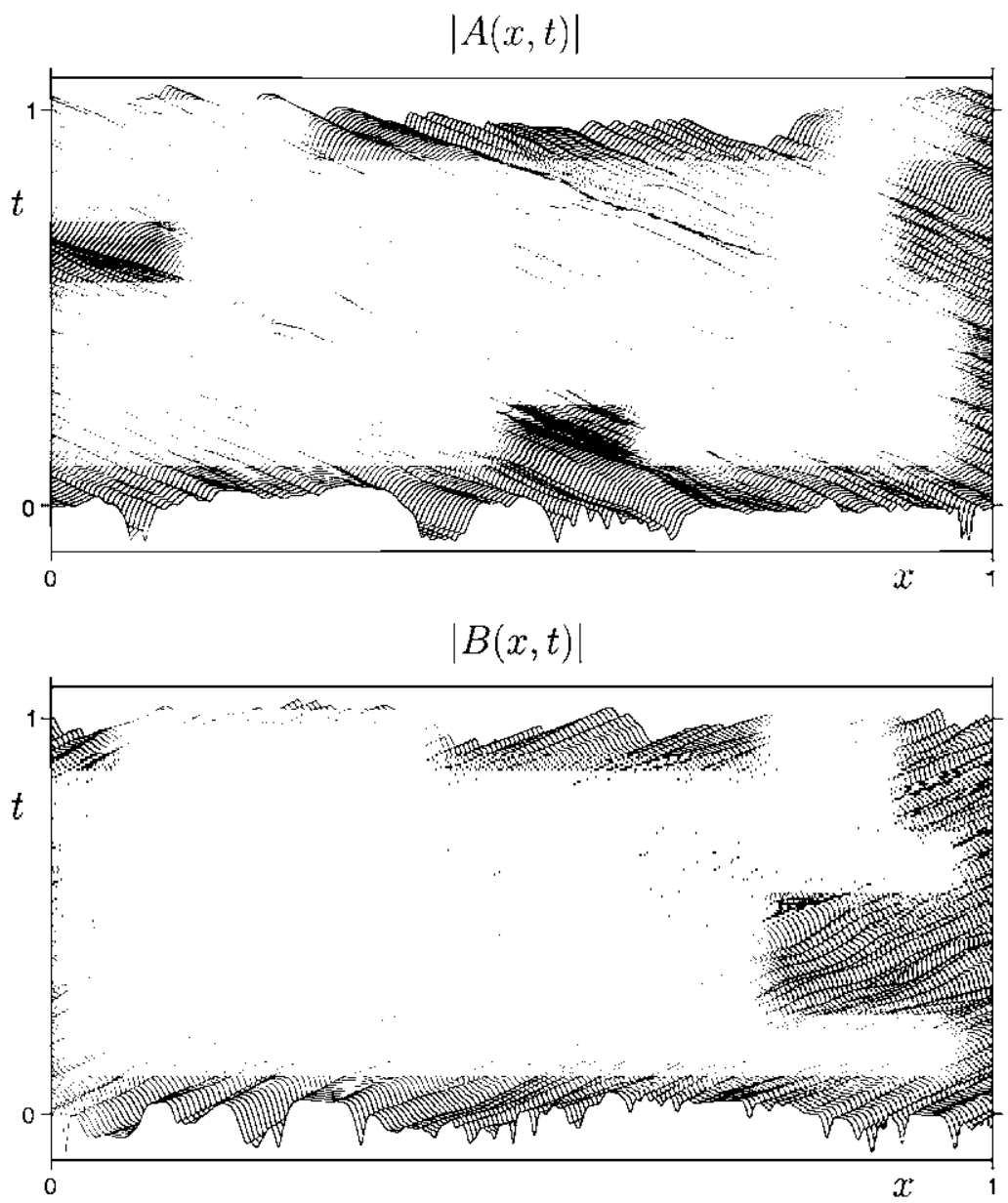

Fig. 13. Space-time representation of the solution of Eqs. (7)-(9) for $\beta=-1, \delta=1, v=1,8=10^{-4}$ and $\mu=4$. 
the hyperbolic description (10)-(12) (Fig. 12c) but the sharp fronts are not produced by the intersection of nearby characteristics, and are insicad due to the appearance of jumps along them. A simple example of this phenomenon is provided by the following, related, model hyperbolic equation

$$
\Lambda_{t}-\Lambda_{x}=-(1+\mathrm{i} v) \Lambda+\mathrm{i}|\Lambda|^{2} \Lambda+\mathrm{i} \gamma .
$$

When integrated along characteristics, this equation becomes an area-contracting ODE, whose solutions converge to the set of steady states. When $v>\sqrt{3}$ and $\gamma_{-} \leq \gamma \leq \gamma_{+}$, where $\gamma_{\mp}=R_{ \pm} \sqrt{1+\left(R_{ \pm}^{2}-v\right)^{2}}$ and $R_{ \pm}^{2}=$ $\left(2 v \pm \sqrt{v^{2}-3}\right) / 3$, there are three such states, say, $\left|\Lambda_{1}\right|<\left|\Lambda_{2}\right|<\left|\Lambda_{3}\right|$, with $\Lambda_{2}$ unstable and $\Lambda_{1}$ and $\Lambda_{3}$ asymptotically stable. The domains of attraction of $A_{1}$ and $A_{2}$ are open sets of the complex plane with a common boundary $\mathcal{A}$ (the unstable manifold of the intermediate steady state). If the initial condition $A(x, 0)$ is such that it crosses $\mathcal{A}$ the solution $A(x, t), t>0$, necessarily develops a front for large $t$ along the characteristic of each intersection point. The presence of weak dispersion thus smooths the solution of the hyperbolic system [17] preventing the formation of infinite gradients and the resulting solutions (Figs. 12a and b) exhibit small dispersive scale oscillations at the front and back of the pulses. Oscillations of this type are typical of dispersive systems (cf. [18]) and are suppressed if the hyperbolic approximation is regularized using diffusion instead of dispersion. Fig. 12c shows an example oblained by adding the terms $\varepsilon A_{x x}$ and $\varepsilon B_{x x}$ to the right-hand sides of Eqs. (10) and (11), respectively, with $\varepsilon=10^{-6}$ and integrating the resulting cquations.

The branch of asymmetric pulses ceases to exist at $\mu \cong 1.95$ where a hystcretic transition to stable SW takes place. In contrast, when the forcing amplitude is increased the symmetric pulse state loses stability and the dispersive scalcs spread all over the domain producing once again complicated spatio-lemporal behavior (Fig. 13).

The dynamics found for the case $\beta=-1 / 3, \delta=1, v=0$ and $\varepsilon=10^{-4}$ (Fig. 7III ${ }^{+}$) are completely similar to those described above for $\beta=-1$.

In the case $\beta=-1, \delta=1, v=0$ ) and $\varepsilon=-10^{-4}$ (Fig. 7IV ${ }^{-}$) the SW are stable if the forcing is below the critical valuc $\mu_{\mathrm{c}} \cong 1.41$ (sec Eqs. (15) and (26)), i.c., if the amplitude of the $\mathrm{SW}$ is below the line $\mathrm{H}_{\mathrm{i}}$ in Fig. $7 \mathrm{IV}^{-}$. Al $\mu=\mu_{\mathrm{c}}$
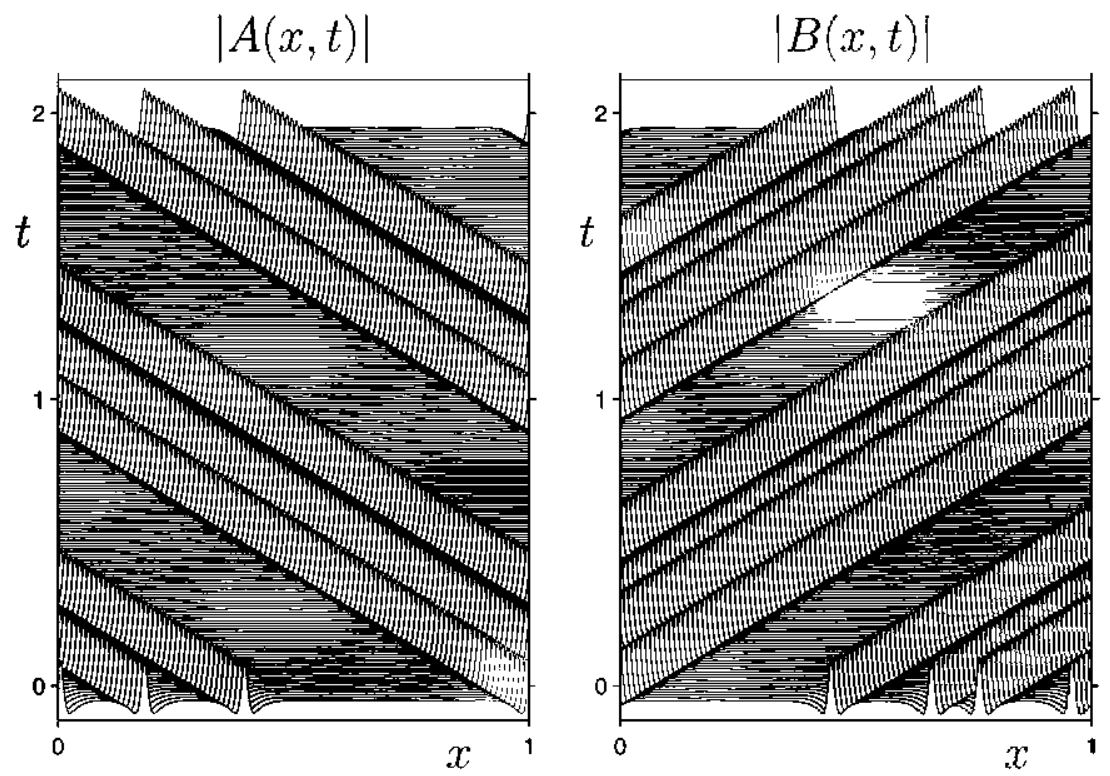

Fig. 14. Space-time representation of the solution of Eqs. (7)-(9) for $\beta=-1, \delta=1, v=0, \varepsilon=-10^{-4}$ and $\mu=1.5$. 
the system undergoes a hysteretic transition to a state consisting of localized narrow counter-propagating (dispersive) pulses that modulate the basic SW state (Fig. 14). Since the primary bifurcation is subcritical the system never enters the region where the hyperbolic approximation applies, despite the large scale of the destabilizing mode. The number and location of the pulses that form depends on the initial conditions and the pattern produced is composed of narrow packets of traveling waves that propagate over the SW wavetrain. These localized solitary waves travel with the group velocity and, at first order, are given by

$$
\begin{aligned}
& A=F(\xi, t)+\cdots, \quad B=B_{0}+\cdots, \quad F_{t}=(-\delta+\mathrm{i} \nu) F+\mu \bar{B}_{0}+\mathrm{i} F\left(\beta|F|^{2}+(1-\beta)\left|B_{0}\right|^{2}\right)-\mathrm{i} F_{\xi \xi}, \\
& F \rightarrow A_{0} \text { as } \xi \rightarrow \pm \infty,
\end{aligned}
$$

where $\xi=(x+t) / \sqrt{|\varepsilon|}$ and $\left(A_{0}, B_{0}\right)$ is the corresponding standing wave. This equation is a damped NLS equation with direct forcing and the solitary waves found in the numerical simulations are essentially the solitons and multisoliton complexes investigated by Barashenkov and collaborators [19-21] but traveling at the group velocity. Note that the solution may consist of different numbers of solitons of width $\sim \sqrt{|\varepsilon|}$ traveling in the positive and negative directions (Fig. 14). These localized solutions coexist with the stable SW when the forcing is reduced
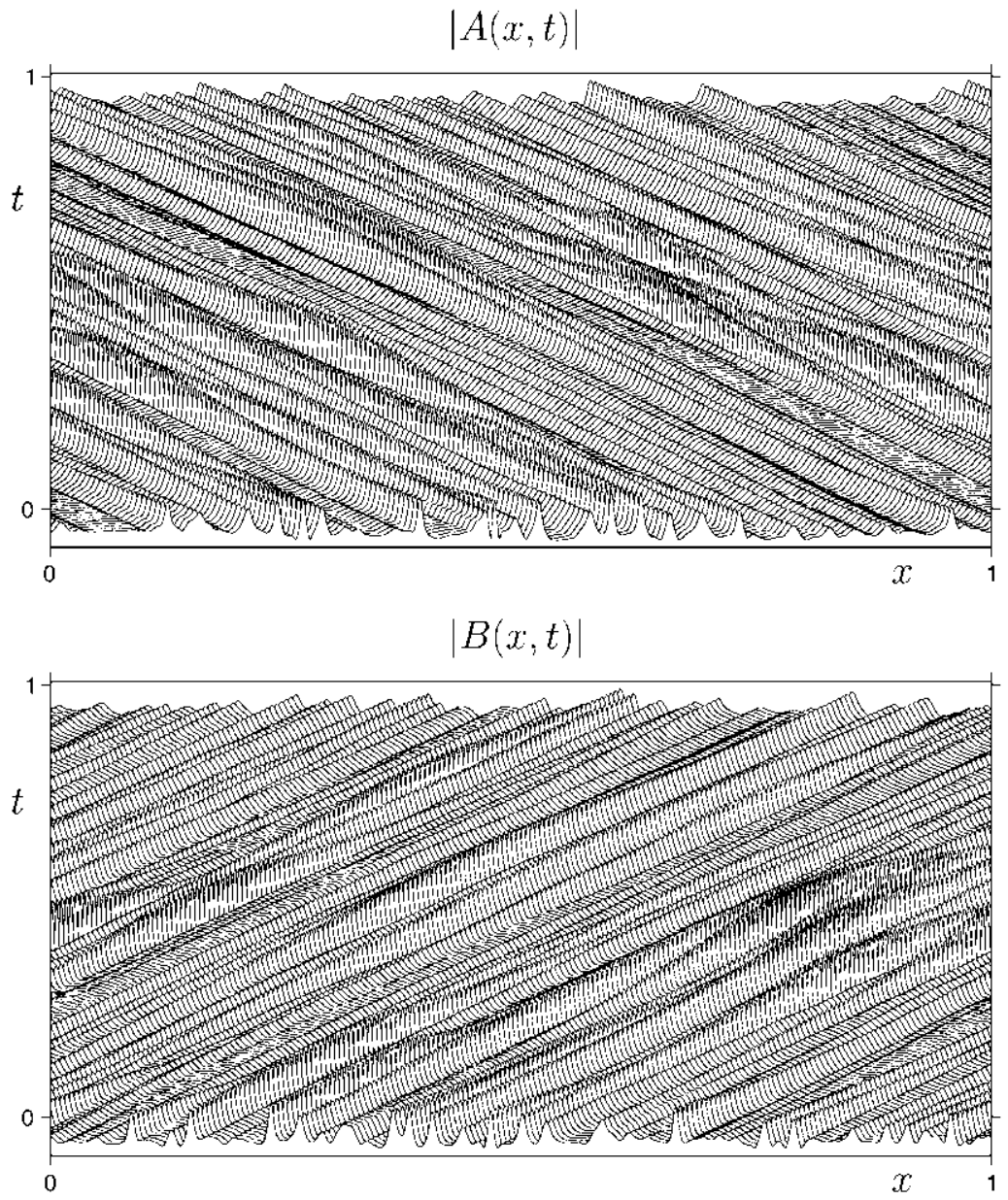

Fig. 15. Space-time representation of the solution of Eqs. $(7)-(9)$ for $\beta=-1, \delta=1, v=0,8=-10^{4}$ and $\mu=4$. 
down to 1.30 while if the forcing is increased they gradually broaden, acquiring additional spatial oscillations at the front and back, before undergoing a Hopf bifurcation that introduces temporal oscillations into their dy namics. For yet higher forcing amplitudes the dispersive scales spread throughout the entire system and the system exhibits spatio-tcmporal chaos (Fig. 15).

Finally, for the case in Fig. 7III-, the SW branch bifurcates supercritically $(v>0)$ and remains stable throughoul.

\section{Concluding remarks}

The weakly nonlinear evolution of counterpropagating, almost conservative, parametrically forced waves in extended annular (or periodic) domains has been investigated. In contrast to systems in which advection is unimportant in the system studied advection at the group velocity dominates the dynamics. When the forcing of the system is sufficiently strong ( $\mu \sim 1 / L$ ) the envelope equations governing the evolution of the system take the form of two coupled, parametrically forced, damped NLS equations with oppositely directed advection and small dispersion, Eqs. (7)-(9). Systems of this type are characterized by two distinct spatial scales both of which can manifest themselves simultaneously whenever the domain is large enough to accommodate them. These scales result from a balance between forcing and advection, and forcing and dispersion, and we have referred to them as the transport and dispersive scales, respectively. In these circumstances the retention of arbitrarily small dispersion may have dramatic consequences for the dynamics of the system, since depending on its sign the presence of dispersion may promote the explosive appearance of dispersive scales.

It should be emphasized that this situation is generic for systems with rellection symmetry admitting propagative dynamics. Indeed, unless one lakes extraordinary pains to minimize advection effects (c.g. by locating codimension-lwo points [22]) advection will always dominate the dy namics near onset. Most extended systems, unless they are too small or too close to onset, will fall in the regime studied here. Such systems include not only the nearly inviscid Faraday system but also a variely of optical systems $[9,10]$.

For such systems the main results are as follows:

- SW locked to the forcing frequency form the first state obtained right after onset, as noted already in [23]. As the forcing is increased these waves lose stability to perturbations on either the transport scale, Eq. (24), or on the dispersive scale, Eq. (27).

- For forcing amplitude and detuning that are not too large and appropriate values of dispersion and the nonlinear coefficient $\beta$ (cases $\mathrm{I}^{-}, \mathrm{II}^{-}$and $\mathrm{IV}^{+}$in Fig. 7), the dynamics of the system are governed by the hyperbolic Eqs. (10)-(12). In these regimes the dispersive scale manifests itself at most locally, and the function of the dispersion is to smooth the solutions of the hyperbolic equations that would otherwise develop shocks.

- If the forcing is strong enough Eqs. (7)-(9) either have uniform SW as the only attractors present (for small values of the nonlinear coefficient $\beta$ and negative dispersion) or develop spatio-temporally complex states with small dispersive scales across the whole domain.

- The behavior of the spatio-temporally complex solutions with dispersive scales resembles that of the NLS equation [16]: the dispersive structures look like depressions (dark solitons) when $\beta \varepsilon<0$ (Figs. 11 and 13) and like humps (bright solitons) when $\beta \varepsilon>0$ (Figs. 9 and 15).

- The dispersive scales appear throughout the domain if $\beta>0$ (Fig. 8) but manifest themselves only locally if $\beta<0$, producing cither dispersive (oscillatory) fronts (and backs) when $\varepsilon>0$ (Fig. 12) or narrow solitary waves when $\varepsilon<0$ (Fig. 14). In the former case bound states of fronts and backs form broad pulses with width that is independent of $\varepsilon$ for small $\varepsilon$. In the latter case the width of the solitary waves scales as $|\varepsilon|^{1 / 2}$ as $\varepsilon \rightarrow 0$, and hence the exact value of $\varepsilon$ remains essential for determining the form of the solution even for small $\varepsilon$. Moreover, in the 
(a)

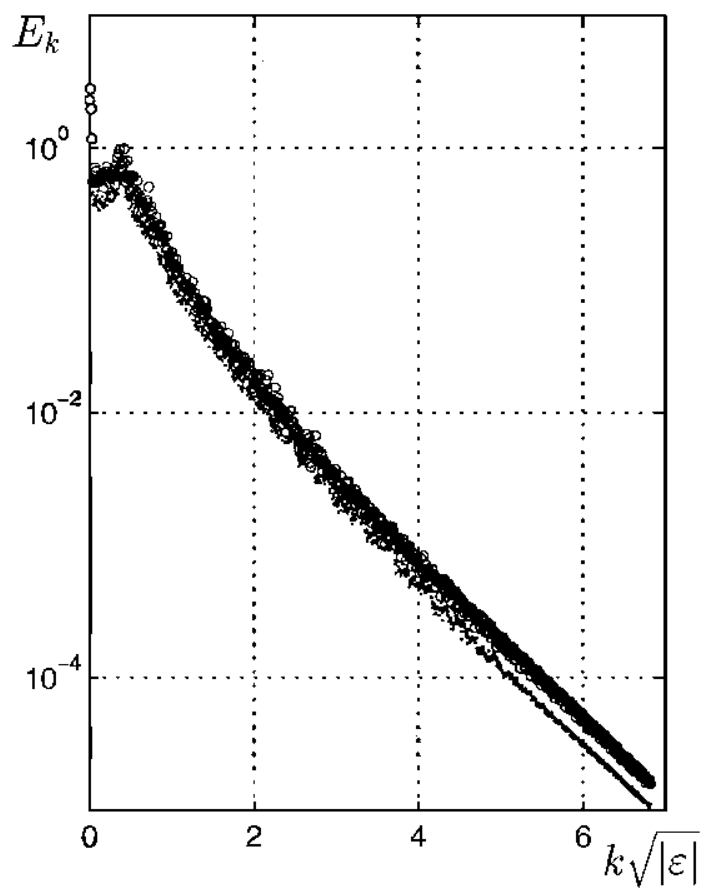

(c)

$E_{k}$

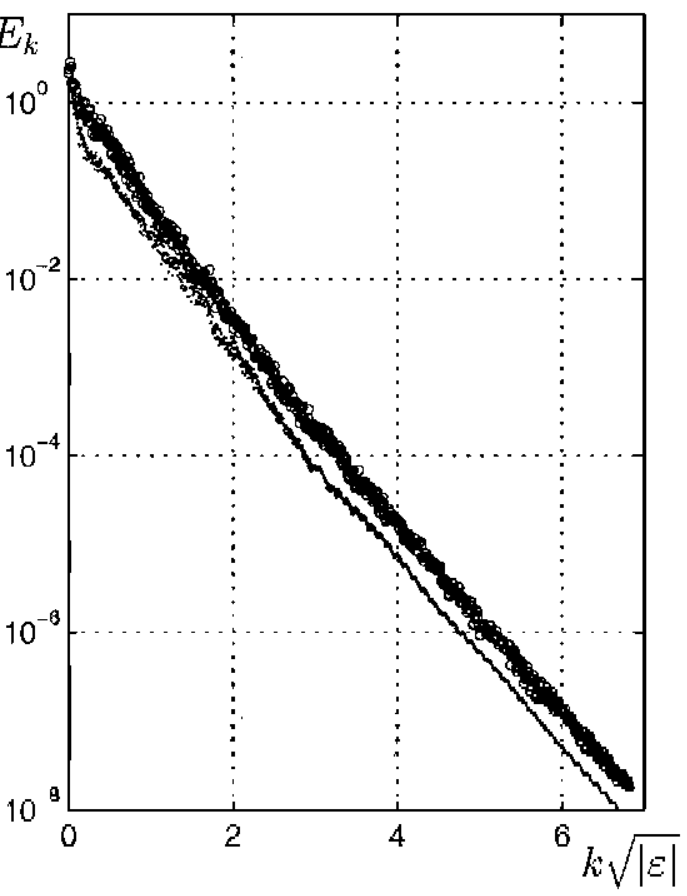

(b)

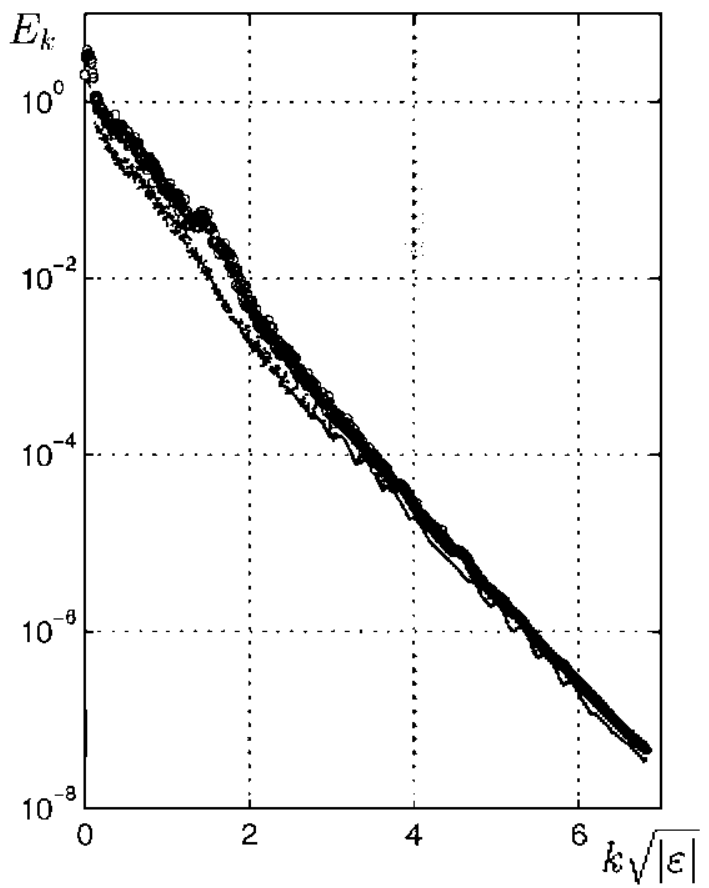

(d)

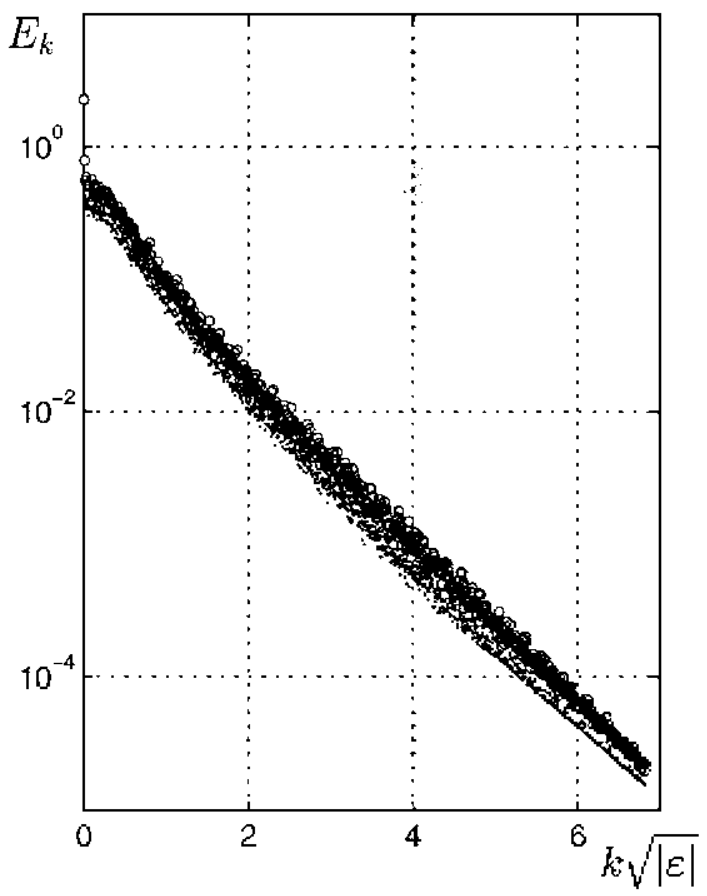

Fig. 16. Maximum spectrum $E_{k}$ for $|\varepsilon|=10^{-1}$ (circles) and $|\varepsilon|=10^{-1} / 4$ (dots) for the cases in Figs. 9 (a), 11 (b), 13 (c) and 15 (d). 
former case the solutions are largely independent of initial conditions, while in the latter the initial conditions determine the number and the location of the solitary waves.

These results apply in the regime in which the transport scale $\ell_{\mathrm{gr}} \sim L$ while the dispersive scale $\ell_{\mathrm{d}} \sim L^{1 / 2} \ll \ell_{\mathrm{g}}$, where $L$ denotes the domain length. This regime contains as a sub-limit ( $\delta \sim \mu \sim \sqrt{|\varepsilon|}$ with the scaling of Eqs. (7)-(9)) the regime $\ell_{d} \sim L$ which is described by simplified albeit non-local coupled NLS equations [12] in which the advection effects are responsible for the presence of the non-local coupling. In this regime $\ell_{\mathrm{g}} \gg L$ and $\ell_{\mathrm{d}} \sim L$ and the dynamics contain only one spatial scale. As a result the complexity encountered is confined to the time domain and considerable progress can be made towards understanding its origin [24]. In the regime discussed in the present paper the presence of two distinct length scales makes similar progress much more difficult.

The results summarized above are deduced from a combination of linear stability theory for spatially uniform SW and numerical simulations of Eqs. (7)-(9). The stability theory identifies the instability that generates dispersive scales but its development requires numerical techniques. It is important to note that the number of Fouricr modes required for the numerical integration of Eqs. (7)-(9) should not be too large since otherwise the long wavelength assumption (2) and (3) can be violated. This is because the parabolic approximation produced by the amplitude equations (dotted line in Fig. 1) to the true dispersion relation generates two possible resonant solutions to the relation $\omega_{0}=\omega(k)$ and one must make sure that only the relevant modes (i.e., those near $k_{0}$ in Fig. 1) are present in the solution of the amplitude Eqs. (7)-(9). In order to avoid such spurious solutions and to ensure that the dispersive scales $(\sim \sqrt{|\varepsilon|})$ are properly represented in our numerical simulations, the following relation must hold between the (rescaled) dispersion parameter $\varepsilon \ll 1$ and the number of Fourier modes in the discretization:

$$
\frac{1}{\sqrt{|\varepsilon|}} \ll N_{\text {Fouricr }} \ll \frac{1}{|\varepsilon|} \text {. }
$$

The above problem does not arise in strongly dissipative systems in which the diffusive terms wipe out high wavenumber modes. In order to be sure that the smallest scales present in our simulations were of order $\sqrt{|\varepsilon|}$ the cases in Figs. 9, 11,13 and 15 were recalculated with dispersion $\varepsilon / 4$ and double resolution. The system (7)-(9) was first integrated from $t=0$ to 50 to eliminate transient dynamics and then, from $t=50$ to 100 , the maximum spectrum of the solutions for $\varepsilon$ and $\varepsilon / 4$ was computed: $E_{k}=\max _{t \in[50,100]}\left\{\left|a^{ \pm k}\right|,\left|b^{ \pm k}\right|\right\}$. The results obtained (shown in Fig. 16) indicate that the maximum spectrum does indeed decay exponentially with $k \sqrt{|\varepsilon|}$ as required.

\section{Acknowledgements}

This work was supported by the National Aeronautics and Space Administration under grant NAG3-2152. The work of CM and JMV was partially supported by the Spanish Dirección General de Investigación under grant BFM 2001-2363.

\section{References}

[1] M. Bondila, I.V. Barashenkov, M.M. Bogdan, Topography of attractors of the parametrically driven nonlinear Sehrödinger equation, Physica D $87(1995) 314-320$.

[2] I.V. Barashenkov, F.V. \%emlyanaya, Stable complexes of parametrically driven, damped nonlinear Schrödinger solitons, Phys. Rev. Istt. 83 (1999) 2568-2571.

[3] X. Wang, Parametrically excited nonlinear waves and their localizations, Physica D 154 (2001) $337-359$.

[4] P.C. Danisls, Finite amplitude two-dimensional convetion in a finite rotating system, Proc. R. Soc. I.ond. A 363 (1978) 195-215.

[5] A.B. Fizerskii, M.I. Rabinovich, V.P. Reutov, l.M. Starobinets, Spatiotemporal ehass in the parametric excitation of a capillary ripple, Sov. Phys. JW"TP 64 (1986) 1228-1236. 
[6] C. Martel, J.M. Vega, l)ynamics of a hyperholie system that applies at the onset of the oscillatory instability, Nonlinearity 11 (1998) $105-142$.

[7] A.B. Ezerskii, V.G. Shekhov, Spatiolemporal modulation of surface waves generated by a uniform fiek, Sov. Pliys. Technol. Phys. 34 (1989) 386-391.

[8] J.M. Vega, E. Knobloch, C. Martel, Nearly inviscid Faraday waves in annular containers of moderately Iarge aspect ratio, Physica D 154 (2001) 313336 .

[9] (i.P. Agrawal, Nonlinear Fiber Optics, Opties and Photonies, Academie Press, New York, 1995.

[10] A.B. Aceves, Optical gap solitons: past, present and future; theory and experiments, Chaos $10(2000) 584-589$.

[11] O. Thual, S. Douady, S. Fauve, Parametric instabilities, in: E. Tirapegui, D. Villaroel (Eds.), Instabilities and Nonecpuilibrium Structures II, Kluwer Academic Publishers, Dordrecht, 1989, pp. 227-237.

[12] C. Martel, E. Knobloch, J.M. Vega, Dynamics of counterpropagating waves in parametrically forced systems, Physica D 137 (2000) 94123.

[1.3] C. Martel, J.M. Vega, Finite size effects near the onset of the oseillatory instability, Nonlinearity 9 (1996) $1129-1171$.

[14] C. Marlel, J.M. Vega, Global stability properties of a hyperbolic system arising in pattern formation, Nonlinear Anal. TMA 29 (1997) $439-460$.

[15] A.C. Newell, Solitons in Mathematies and Physies, Society for Industrial and Applied Mathematics, Philadelphia, PA, 1985

[16] P.G. Drazin, R.S. Johnson, Solitons: An Introduction, Cambridge Texts in Applied Mathematics, Cambridge University Press, Cambridge, 1993.

[17] P.I). Lax, C.D. I evermore, S. Venakides, The generation and propagation of oseillations in dispersive IVPs and their limiting behavior, in: T. Fokas, V.E. Zakharov (Eds.), Important Developments in Soliton Theory 1980-1990, Springer, Berlin, 1993, pp. $205-241$.

[18] A. Spina, J. Toomre, E. Knobloch, Conflned states in large aspect ratio thermosolutal convection, Phys. Rev. E 57 (1998) $524-545$.

[19] I.V. Barashenkov, Y.S. Sminnov, Existence and stability chart for the ac-driven, damped nonlinear Sclrödinger solitons, Phys. Rev. E 54 (1996) 57075725 .

[20] I.V. Barashenkov, Y.S. Smirnov, N.V. Alexeeva, Bifurcation to multisoliton complexes in the ac-driven, damped nonlinear Sehrödinger equation, Phys. Rev. F, 57 (1998) 2350-5764.

[21] I.V. Barashenkov, E.V. Zemlyanaya, Existence threshold for the ac-driven damped nonlinear Schrödinger solitons, Physica D 132 (1999) $363-372$.

[22] H. Riecke, L. Kramer, The stability of standing waves with small group velocity, Physica D 137 (2000) 124142.

[23] H.Riecke, J.D. Crawford, E. Knobloch, Time-modulated oscillatory convection, Phys. Rev. Lett. 61 (1988) 19421945.

[24] M. Higuera, J. Porter, F. Knobloch, Heteroclinie dynamies in the nonlocal parametrieally driven nonlinear Schrödinger equation, Physica D $162(2002) 155-187$. 